\title{
Influence of Parameters on vibrations for Centrifugal Pump Based on the Modal Dynamics Method
}

\author{
Guofang Nan ${ }^{1, a}$, Yuanlu Zhang ${ }^{2, b}$ \\ ${ }^{1}$ School of energy and power engineering, University of shanghai for science and technology, \\ Shanghai 200093, China; \\ ${ }^{2}$ School of energy and power engineering, University of shanghai for science and technology, \\ Shanghai 200093, China; \\ angf@usst.edu.cn, bhangyuanluusst@163.com
}

Keywords: parametric analysis, exit installation angle, blade number, modal dynamics.

\begin{abstract}
The influence of the parameters on the vibrations for a centrifugal pump is investigated under the consideration of the fluid excitation force in this paper. The responses of the centrifugal pump during the operation were mostly generated by the fluid excitation force. It is difficult to simulate accurately and make prediction of this coupling vibration in a rigorous analytical method. The finite element model was established in this article with consideration of the effect of the fluid excitation. Based on the modal dynamics method, the analysis is carried out to study on the effect of the parameters such as the flow rate, the blade number, the exit installation angle and the outside diameter of impeller on the frequency-response curves. The results show that there exists an optimal blade number value for a better effect of vibration reduction; the design of the outside diameter should consider the response to get a better damping. The research work is meaningful for the parametric designation and the safe check for the centrifugal pumps.
\end{abstract}

\section{Introduction}

The centrifugal pump is a kind of rotating fluid machinery. The working fluid obtains the energy of the rotating blade during the operation; then the working fluid exerts an unsteady excitation pressure on the body of pump and the shaft of rotation. This excitation pressure produces a coupled vibration of sound field within the pump body and structural responses. The vibration and noise propagate outward along the pipeline. The mechanical noise is directly associated with the pump, for example from the bearings and from the sealing elements. However the mechanical noise is not notable unless there exists mechanical fault. The fluid dynamic noise mainly derives from the interior of the centrifugal pump shell and it is directly associated with the unsteady pulsating flow within the pump body. This type of vibration and noise have an effect on the performance of the machinery system. Accordingly, the research on the vibrational characteristic for the centrifugal pump is significant for the engineering application.

During the last decades, much progress has been made about the vibration of the centrifugal pump. C.G.Rodriguez [1] et. al carries out a theoretical analysis to predict and explain in a qualitative way the frequencies and amplitudes for the rotor stator interaction in a centrifugal pump turbine. A successful corrective action is proposed as a consequence of this new interpretation. Y.Y. Jiang et al [2] reports on reports on a full-scale structural simulation of flow-induced mechanical vibrations and noise in a 5-stage centrifugal pump. They clarified the mechanisms of the resonant vibration and the noise generation and propagation, which can then be used for noise reduction. Andreas Lucius et al [3] researched on the velocity fluctuations during rotating stall of a centrifugal pump in order to determine their importance for excitation of structural vibrations. Lohasz, $M$ et. al [4] and Byskov, $R$ et.al [5] applied the large eddy simulation in the field of turbomachinery to solve more details of the flow field and restricted to low Reynolds numbers. In addition, the hybrid methods [6-9] like detached eddy simulation are used increasingly. Many researchers [10-14] studied the fault diagnoses of the centrifugal pump or rotational machinery. Zhou Yunlong and Zhao Peng [15] proposed a new fault 
diagnose method for the nonlinear and non-stationary characteristics of the vibration signals of centrifugal pump based on complexity feature of Empirical Mode Decomposition (EMD). While V. Muralidharana et al [16] carried out an investigation of fault diagnose for the monoblock centrifugal pump using the support vector machine (SVM) and artificial neural networks were employed for continuous monitoring and fault diagnosis. Even though the mass unbalance is not be eliminated completely, the dynamic balance technology is applied to decrease the influence of the mass unbalance for a minor excitation force. For the rotor misalignment, the periodic excitation force can be effectively decreased if the flexible connections are applied to the junctions of the two rotors. However, the unsteady fluid excitation is the main causation of the vibration and noise and it has not been eliminated effectively at present. Therefore, the construction of the centrifugal pump model and the study of the vibrational characteristics are essential for the theory and engineering application. Dynamic characteristics of centrifugal pump Induced by the fluid excitation force have been studied rarely in the published articles, this research will be reported in this paper.

\section{Modelling and calculation}

Importing the geometry model into the Abaqus and then meshing the components by using the type of C3D4 lead to the analytical model. The different types of element and dimension are used for the different parts in order to obtain the fine geometry features. The meshing quality of the elements is checked to get a good quality and the parts will be remeshed if their quality does not meet the requirements until the quality is good enough for calculation. The parameters used are as follows: Modulus of elasticity $E=210 \mathrm{GPa}$, density $\rho=7850 \mathrm{Kg} / \mathrm{m}^{3}$, Poisson ratio $\mu=0.3$. After calculating the free modal of the components and comparing the results with that of experiments and they agree well.The connection between the two components in centrifugal pump mainly includes the bolting, the shaft coupling and so on. The springs with the different stiffness values are applied to simulate the connections in this paper. The absorber on the frame, the connectors between the inlet/outlet pipes with the exterior are also simulated to be the springs with a specific stiffness values, and the type of springs is connect point to ground. The spring are built with the different types and stiffness with consideration of the stiffness in three components in order to be closer to reality.

The modal dynamics method is adopted in this paper to calculate the responses. This method applies the modal superposition method to solve the transient responses of the linear system. The characteristic modes should be extracted before this method based on the characteristic modes is applied. The method is applied to make the time-domain analysis of a linear system, where the excitation is a function of time and there is an assumption that the amplitude in every incremental time step is linear under the determined excitation. In this paper, the excitation force is the fluid excition force which was obtained by the numerical simulation. This excitation force is exerted on the analytical model to submit calculation.

\section{Results and analysis}

The response on the volute under the fluid excitation will be investigated in this section. The constrained modes of the centrifugal pump which are connected with the inlet/outlet pipe are calculated before the responses are calculated. Appling the Spring (connect point to ground), the feet of the machine, the inlet and the outlet are joined by Springs to the ground. The constrained modes are calculated by using the Lanczos Method; the results are seen in Table 1 . The analytical point on the volute $\mathrm{A}$ is selected as the analytical point of the centrifugal pump, as seen in Figure 1.

In order to study the effect of the blade number, the impeller outside diameter, the exit installation angle on the response of the analytical point, the number of the impeller is selected as: 4 blades, 5 blades, 6 blades, 7 blades and 8 blades, respectively; the outside diameter of the impeller is selected as: $170 \mathrm{~mm}, 171 \mathrm{~mm}, 172 \mathrm{~mm}, 173 \mathrm{~mm}$ and $174 \mathrm{~mm}$, respectively; the exit installation angle is $20^{\circ}$, $23^{\circ}, 27^{\circ}$ and $35^{\circ}$, respectively. Then, the response of the analytical point is extracted for analysis. The 
influence of the flow rate, the blade number, the exit installation angle and the outside diameter on the vibrations will be reported in the next sections.

Table 1 The main constrained modes (constrained on the inlet/outlet and the feet of the machine)

\begin{tabular}{cc}
\hline Order & Frequency $(\mathrm{Hz})$ \\
\hline 1 & 128.18 \\
2 & 154.28 \\
3 & 184.43 \\
4 & 282.95 \\
5 & 301.85 \\
6 & 343.89 \\
7 & 363.71 \\
8 & 384.88 \\
\hline
\end{tabular}

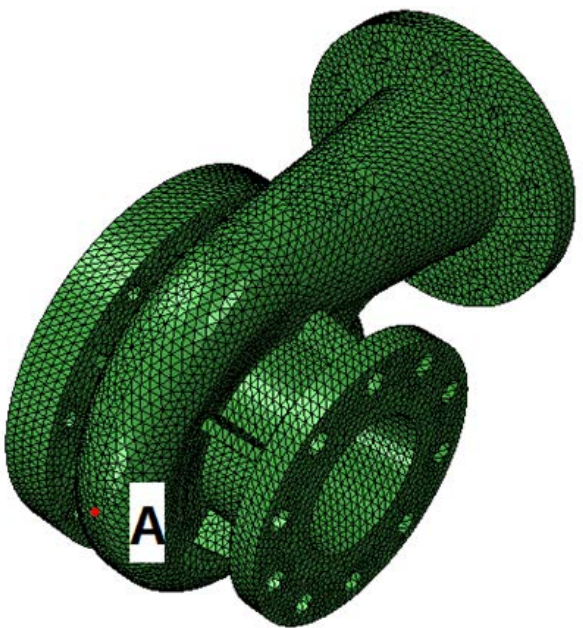

Fig. 1 The analytical model and the analytical point A of the centrifugal pump

\section{The flow rate}

The flow rate is the liquid quantity delivered in unit time; it is the fundamental parameter of the performance for the centrifugal pump. The influence of the flow rate on the vibration is investigated in this section. Figure 2 shows the FR (Frequency Response) curves for the analytical point A with the different flow rate 0.6Q, 0.8Q, 1.2Q and 1.4Q. It can be seen from Figure 2 that the acceleration responses of the analytical point for the centrifugal pump decreases with the increasing of the flow rate; the different flow rates excite the same resonant frequency $291 \mathrm{~Hz}$.

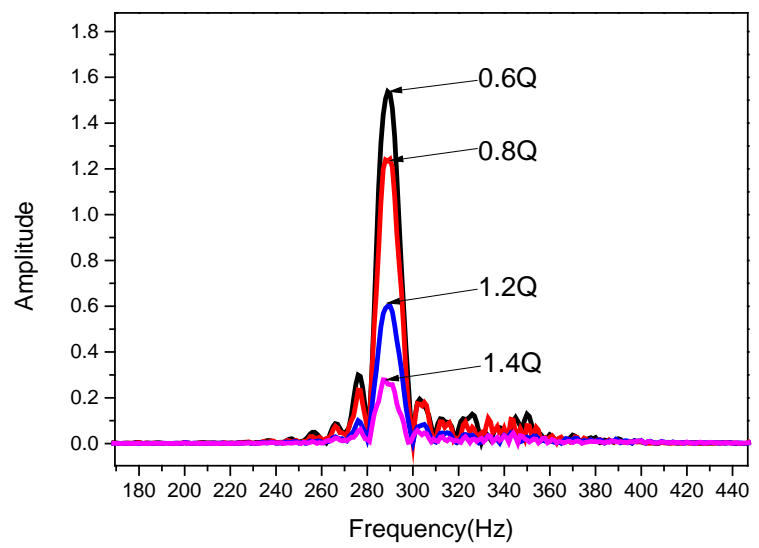

Fig. 2 The influence of the flow rate on the FR curves 


\section{The blade number}

The blade number is the important parameter of the centrifugal pump. Figure 3 shows the FR curves for the analytical point with the different blade number 4-blade, 5-blade, 6-blade and 7-blade. It can be seen from Figure 3 that the max acceleration responses of the analytical point is for the 5-blade, while the minimal acceleration response is for the 6-blade; the frequency multiplication $193 \mathrm{~Hz}, 242 \mathrm{~Hz}, 290 \mathrm{~Hz}$ and $338 \mathrm{~Hz}$, which are the multiplications of the shaft frequency $48.3 \mathrm{~Hz}$ (rotating speed is $2900 \mathrm{r} / \mathrm{min}$ ), appear for the blade number 4-blade, 5-blade, 6-blade and 7-blade, respectively; the frequency of the response peak is $386 \mathrm{~Hz}, 296 \mathrm{~Hz}, 290 \mathrm{~Hz}, 340 \mathrm{~Hz}$ for the 4-blade, 5-blade, 6-blade and 7-blade, respectively. Therefore, the optimal number of the blade is 5-blade only under the consideration of the vibration reduction. The resonant frequency and the reducing vibration should be considered comprehensively in selecting the blade number.

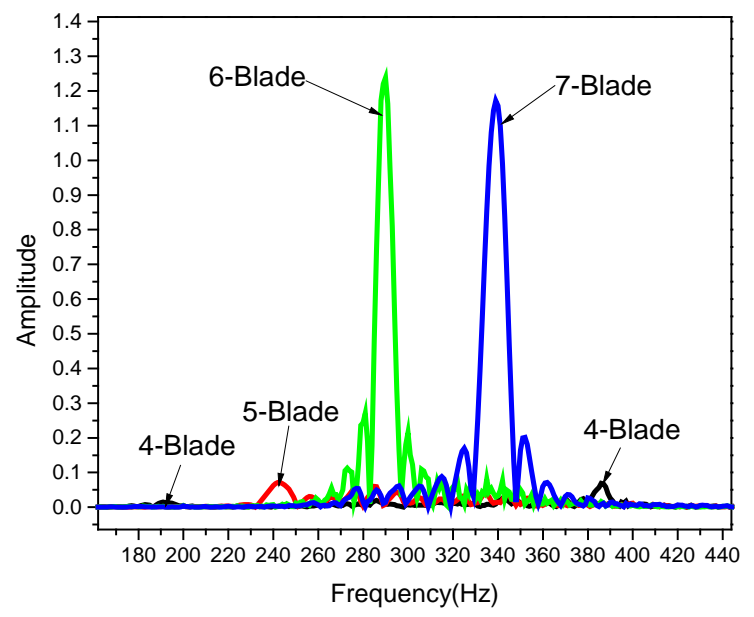

Fig. 3 The influence of the blade number on the FR curves

\section{The exit installation angle}

The exit installation angle is an essential parameter of the centrifugal pump. The exit installation angles are selected as $20^{\circ}, 23^{\circ}, 27^{\circ}, 30^{\circ}$ and $35^{\circ}$. The influence of them on the vibrations is shown in Figure 4. It can be seen from Figure 4 that the different angles have the same resonant frequency $290 \mathrm{~Hz}$; and the maximal amplitude is for the $30^{\circ}$ which is nearly equal to the amplitude for $35^{\circ}$; while the minimal amplitude is for $20^{\circ}$.

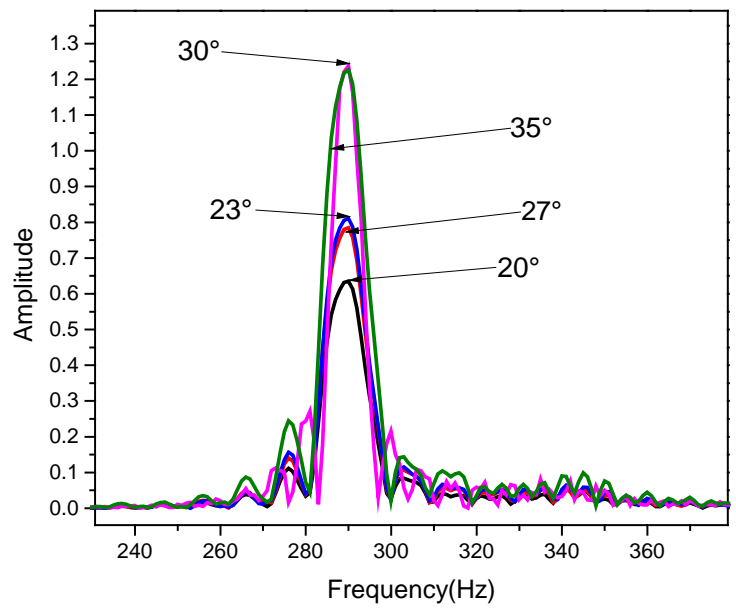


Fig. 4 The influence of the exit installation angle on the FR curves

\section{The outside diameter}

The outside diameter of the impeller for the centrifugal pump has effect on the dynamic characteristics of the system. The outside diameters of the impeller are selected as $170 \mathrm{~mm}, 171 \mathrm{~mm}$, $172 \mathrm{~mm}, 173 \mathrm{~mm}$ and $174 \mathrm{~mm}$. The influence of them on the vibrations is shown in Figure 5. It can be seen from Figure 5 that the different outside diameters have the same resonant frequency $290 \mathrm{~Hz}$; and the maximal amplitude of the response is for the $174 \mathrm{~mm}$ while the minimal amplitude is for $170 \mathrm{~mm}$. Accordingly, the design of the outside diameter should consider the response to get a better damping; in this calculation example, the $170 \mathrm{~mm}$ is the better selection only from the vibration reduction.

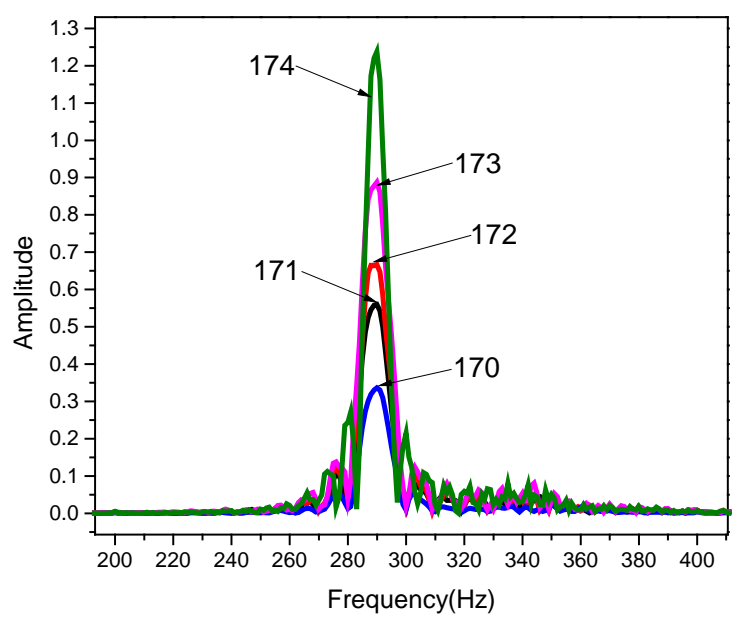

Fig. 5 The influence of the outside diameter on the FR curves

\section{Summary}

The numerial model of a centrifugal pump was established under the consideration of the fluid excitation force in this paper. Based on the modal dynamics method, the analysis was carried out to study the effect of the parameters such as the flow rate, the blade number, the exit installation angle and the impeller outside diameter on the dynamic characteristics. The main conclusions are as follows:

$>$ The responses of the centrifugal pump under the fluid excitation force decreases with the increasing of the flow rate; the different flow rates excite the same resonant frequency $291 \mathrm{~Hz}$. The optimal number of the blade is 5-blade from the perspective of the vibration reduction. The resonant frequency and the reducing vibration should be considered comprehensively in selecting the blade number.

$>$ The different exit installation angles have the same resonant frequency $290 \mathrm{~Hz}$; and the maximal amplitude is for the $30^{\circ}$ while the minimal amplitude is for $20^{\circ}$. The design of the outside diameter should consider the response to get a better damping; and in this calculation example, the $170 \mathrm{~mm}$ is the better selection only from the vibration reduction. It is prudent and comprehensive to design the parameters of the centrifugal pump.

\section{Acknowledgement}

The authors gratefully acknowledge the financial support of the National Science Foundation of China (Granted number: 51305267). 


\section{References}

[1] Odriguez C G, Egusquiza E, Santos I F. Frequencies in the vibration induced by the rotor stator interaction in a centrifugal pump turbine[J]. Journal of Fluids Engineering, 2007, 129(11):1428-1435.

[2] Y.Y. Jiang_, S. Yoshimura, R. Imai, H. Katsura, T. Yoshida, C. Kato, Quantitative evaluation of flow-induced structural vibration and noise in turbomachinery by full-scale weakly coupled simulation[J] Journal of Fluids and Structures 23 (2007) 531-544

[3] Andreas Lucius, Gunther Brenner, Numerical simulation and evaluation of velocity fluctuations during rotating stall of a centrifugal pump[J] Journal of Fluids Engineering, 2011; 133(8):081102-081102-8.

[4] Lohasz, M. M., Nagy, L., and Wurm, H., 2009, "LES of the Transitional Flow in a Miniature Centrifugal Pump,” in Proceedings of the 14th Conference on Modelling Fluid Flow.

[5] Byskov, R., Jacobsen, C., and Pedersen, N., 2003, "Flow in a Centrifugal Pump Impeller at Design and Off-Design Conditions - Part II: Large Eddy Simulations,” J. Fluids Eng., 125, pp. 73-83.

[6] Feng, J., Benra, F.-K., and Dohmen, H., 2009, "Unsteady Flow Visualization at Part-Load Conditions of a Radial Diffuser Pump: By PIV and CFD,” J. Visualization, 12(1), pp. 65-72.

[7] Martini, P., Schulz, A., Bauer, H.-J., and Whitney, C., 2006, "Detached Eddy Simulation of Film Cooling Performance on the Trailing Edge Cutback of Gas Turbine Airfoils,” ASME J. Turbomach., 128(2), pp. 292-299

[8] Iwakiri, K., Furukawa, M., Ibaraki, S., and Tomita, I., 2009, "Unsteady and Three-Dimensional Flow Phenomena in a Transonic Centrifugal Compressor Impeller at Rotating Stall,” Proceedings of the ASME Turbo Expo, 7(B), pp. 1611-1622.

[9] Lucius, A., and Brenner, G., 2010, "Unsteady CFD Simulations of a Pump in Part Load Conditions Using Scale-Adaptive Simulation,” Int. J. Heat Fluid Flow, 31, pp. 1113-1118.

[10]N.R. Sakthivel, Binoy.B.Nair, V. Sugumaran, Soft computing approach to fault diagnosis of centrifugal pump, Applied Soft Computing, Volume 12, Issue 5, May 2012, Pages 1574-1581

[11]N.R. Sakthivel, V. Sugumaran, S. Babudevasenapati, Vibration based fault diagnosis of monoblock centrifugal pump using decision tree, Expert Systems with Applications, Volume 37, Issue 6, June 2010, Pages 4040-4049

[12] Shengwei Wang, Jingtan Cui, Sensor-fault detection, diagnosis and estimation for centrifugal chiller systems using principal-component analysis method, Applied Energy, Volume 82, Issue 3, November 2005, Pages 197-213

[13] Yang Zhao, Shengwei Wang, Fu Xiao, A statistical fault detection and diagnosis method for centrifugal chillers based on exponentially-weighted moving average control charts and support vector regression, Applied Thermal Engineering, Volume 51, Issues 1-2, March 2013, Pages 560-572

[14] N.R. Sakthivel, Binoy B. Nair, M. Elangovan , V. Sugumaran , S. Saravanmurugan, Comparison of dimensionality reduction techniques for the fault diagnosis of mono block centrifugal pump using vibration signals[J] Engineering Science and Technology, an International Journal, 17 (2014) 30-38

[15]Zhou Yunlong, Zhao Peng, Vibration Fault Diagnosis Method of Centrifugal Pump Based on EMD Complexity Feature and Least Square Support Vector Machine [J] Energy Procedia 17 ( 2012 ) 939-945

[16] V. Muralidharana,, V. Sugumaranb, V. Indirac, Fault diagnosis of monoblock centrifugal pump using SVM [J], Engineering Science and Technology, an International Journal 17 (2014) 152-157. 